\title{
A prática do núcleo de apoio à saúde da família do Recife no enfrentamento à pandemia COVID-19
}

\author{
Maria Angélica Bezerra de Oliveira, Laís de Souza Monteiro, Rebeca de Castro Oliveira, \\ Tarcilla Sousa Moreira, Ana Carolina Fonseca Marques, Úrsula Maria de Araújo Silva, \\ Nadvânia Albuquerque Oliveira, Gladys Fernanda Coelho Pereira, Ana Claúdia Serra Silva, \\ Renata Morais de Santana
}

\section{RESUMO}

Este artigo tem por objetivo relatar a experiência de reorganização do processo de trabalho do Núcleo de Apoio a Saúde da Família (NASF) na Atenção Primária a Saúde (APS) visando a pandemia da COVID-19 no município do Recife, Pernambuco. Adequando-se ao contexto de cada território e demandas específicas das equipes de Saúde da Família (eSF), as atividades e ações do NASF foram gradativamente reformuladas para manter a continuidade do cuidado no território frente a pandemia da COVID-19. As atividades realizadas são apresentadas no presente artigo em três eixos: atividades ligadas diretamente aos usuários, suporte as eSF e seus profissionais e as ações nos territórios. Consideramos que as possibilidades de atuação do NASF ampliaram a capacidade de visão da comunidade e o desenvolvimento de habilidades para o enfrentamento deste desafio e outros tantos que virão no âmbito da APS.

Palavras-chave: Serviços de saúde; Atenção Primária à Saúde; Estratégia Saúde da Família; Atitudes e Práticas em Saúde; COVID-19.

\section{ABSTRACT}

This article aims to report the experience of reorganizing the work process of the Family Health Support Center (NASF) in Primary Health Care (APS) aiming at the COVID-19 pandemic in the city of Recife, Pernambuco. Adapting to the context of each territory and the specific demands of the Family Health teams (eSF), NASF's activities and actions were gradually reformulated to maintain continuity of care in the territory in the face of the COVID-19 pandemic. The activities carried out are presented in this article in three axes: activities directly linked to users, support for their professionals and actions in the territories. We believe that NASF amplie's possibilities of action have expanded the community's vision capacity and the development of skills to face this challenge and many others that will come within the scope of APS.

Keywords: Health Services; Primary Health Care; Family Health Strategy; Health Knowledge, Attitudes, Practice; Coronavirus Infections.
Revista da Rede APS 2020

Publicada em: 09/06/2020

DOI:10.14295/aps.v2i2.96

Maria Angélica B. de Oliveira Laís de Souza Monteiro Rebeca de Castro Oliveira Tarcilla Sousa Moreira Ana Carolina Fonseca Marques Úrsula Maria A. Silva Nadvânia A. Oliveira Gladys Fernanda C. Pereira Ana Cláudia S. Silva Renata de Morais Santana (Prefeitura da Cidade do Recife, Recife, PE, Brasil)

Correspondência para: Maria Angélica B. de Oliveira angelica.mabo@gmail.com

Laís de Souza Monteiro lais.souza.monteiro@gmail.com

Rebeca de Castro Oliveira rebeca.castrooliveira@gmail.co $\underline{m}$ 


\section{INTRODUÇÃO}

O Núcleo de Apoio à Saúde da Família (NASF) é definido pela Política Nacional de Atenção Básica (PNAB, 2017), como uma equipe de apoio às equipes de Saúde da Família (eSF) e Atenção Básica, composta por profissionais de diferentes campos de conhecimento, que devem atuar de maneira integrada e apoiando às eSF compartilhando as práticas e saberes em saúde nos território.

Há 10 anos o NASF foi implantado no município do Recife, Pernambuco, sendo as equipes compostas por sete (07) categorias profissionais: fisioterapeuta, fonoaudiólogo, psicólogo, terapeuta ocupacional, farmacêutico, nutricionista e assistente social. Atualmente, o município tem 134 profissionais, organizados em 20 equipes distribuídas nos oito Distritos Sanitários (DS) que organizam a rede municipal de saúde.

Os NASF se constituem como serviços dependentes, tendo o acesso para atendimento individual ou coletivo regulados pelas eSF apoiadas. As equipes devem atuar de forma integrada à Rede de Atenção à Saúde e seus diversos pontos de atenção, além de outros equipamentos sociais públicos/privados, redes sociais e comunitárias (PNAB, 2017).

A partir das demandas identificadas, as equipes conjuntamente, discutem casos e planejam ações em reunião de equipe. O plano terapêutico é discutido e, à luz do Apoio Matricial (CAMPOS, 2007), poderá ser executado, estruturalmente, pela (1) Dimensão clínico-assistencial: Atendimento individual ou compartilhado com eSF; Atendimento em grupo (educativos e/ou terapêuticos); Ações de educação em saúde; dentre outras, e/ou na (2) Dimensão Técnico-pedagógica: Reunião de equipe; discussão de caso clínico; construção de Projeto Terapêutico Singular (PTS); Educação Permanente entre profissionais da eNASF e para eSF (matriciamento); Articulação intra e intersetorial, dentre outras.
O recente surto de COVID-19 gerou uma pandemia mundial. Até o dia 25 de maio do corrente ano, o Estado de Pernambuco ocupou o sexto lugar em números de casos (29.919) confirmados de COVID-19 (BRASIL, 2020) e, Recife totalizou 13.712 casos confirmados para COVID-19, e destes 902 evoluíram para o óbito e 4.532 se recuperaram da doença (RECIFE, 2020a). Esses dados sinalizaram a necessidade de reorganização do processo de trabalho da Atenção Primária à Saúde (APS), incluindo o NASF enquanto participante deste cenário.

Assim, este artigo visa relatar a experiência de reorganização do processo de trabalho do NASF na APS tendo em vista a pandemia da COVID-19 no município do Recife, Pernambuco.

\section{(RE)ORGANIZAÇÃO DO PROCESSO DE TRABALHO NASF}

Desde março do corrente ano, a APS do Recife foi tomada pela pandemia da COVID-19 mobilizando o NASF a refletir sobre o processo de trabalho já consolidado. Coube ao coletivo de gestores e trabalhadores, fazer as seguintes perguntas: Como podemos continuar apoiando as eSF e comunitários neste momento de Pandemia?

Assim, as atividades do NASF com as equipes apoiadas passaram por reformulações para se adequar ao contexto de cada território e as demandas específicas de cada eSF. Enquanto equipe de APS foi necessária a expedição de uma Nota Técnica (NT) instrutiva versando sobre o contexto do processo de trabalho do NASF na Pandemia, estando esse alinhado com o processo de trabalho das eSF apoiadas. Neste momento, só gestores contribuíram com este documento (RECIFE, 2020b; RECIFE, 2020c).

Após essa etapa, algumas unidades de cobertura NASF, foram destacadas para receberem, exclusivamente, pacientes com COVID-19, servindo de referência para toda a microrregião (Recife, 2020d), bem como, a contribuição do 
relato das práticas dos profissionais NASF, nos levou a necessidade de novas alterações no fazer, trazendo adaptações e outras possibilidades de atuação em um constante movimento de reinvenção, pois a cada alteração do processo de trabalho das eSF, necessitaria de um processo de adaptação do NASF. Assim, a NT 02/2020 aponta para possibilidades de atuação das práticas de forma presencial e remota objetivando reduzir aglomeração nas Unidades de Saúde e maior proteção aos profissionais de saúde e usuários (RECIFE, 2020e).

$\mathrm{Na}$ versão 3 do Protocolo de Assistência e manejo Clínico do Novo Coronavírus na APS do Recife (RECIFE, 2020f), o processo de trabalho do NASF já consta acoplado as atividades APS do município, reforçando o papel fundamento do NASF no enfrentamento da pandemia.

Figura 01 - Linha do tempo das normativas do processo de trabalho da APS em recife, Pernambuco.

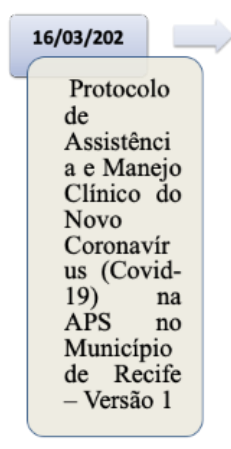

\begin{tabular}{|lr|}
\hline 18/03/2020 \\
\hline Nota & Técnica \\
$\mathrm{N}^{\circ}$ & $01 / 2020$ \\
Processo & de \\
trabalho & do \\
Nasf & no \\
contexto & de \\
enfrentamento \\
da emergência \\
em & saúde \\
pública & \\
decorrente do & do \\
novo & \\
coronavírus \\
(COVID-19) \\
\hline
\end{tabular}

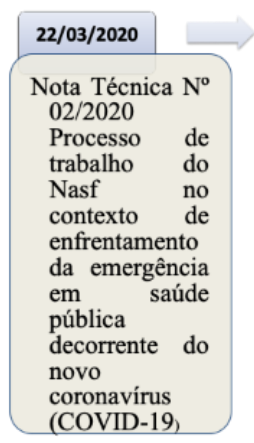

\begin{tabular}{|l|}
\hline 29/03/2020 \\
Protocolo de \\
Assistência e \\
Manejo do \\
Clínico do \\
Novo \\
Coronavírus \\
(Covid-19) \\
na APS no \\
Município \\
de Recife - \\
Versão 2 \\
\end{tabular}
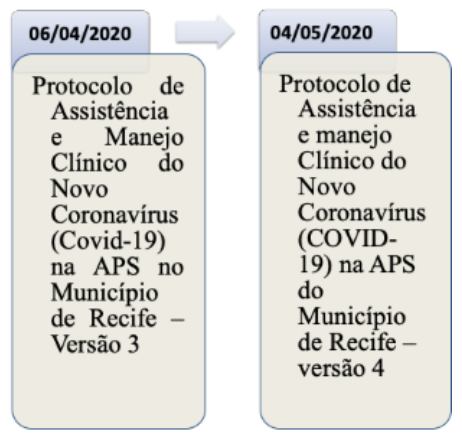

Fonte: elaboração própria.

\section{VIVÊNCIAS E EXPERIÊNCIAS DO NASF FRENTE À COVID-19}

Em Recife o NASF tem uma cobertura de 59,5\% das equipes de saúde da família do município. Cada equipe NASF apoia entre oito e nove eSF, entretanto, com as estratégias de enfrentamento à COVID e as medidas de proteção ao contágio, os profissionais concentraram suas atividades nas USF onde eles tem uma estrutura melhor de organização e onde tem espaço físico para guarda de material, denominado de ponto de apoio, designando um técnico de referência para as demais equipes apoiadas.

Deste modo, as equipes do NASF Recife foram gradativamente reformulando suas práticas e desenvolvendo novas ações para o enfrentamento à COVID-19. Por meio de uma organização interna mais efetiva atuaram de modo a manter a continuidade do cuidado no território e desenvolveram diferentes possibilidades de atuação, no contexto da pandemia. Assim, traremos as atividades realizadas em três eixos: atividades ligadas diretamente aos usuários, suporte as eSF e seus profissionais e as ações nos territórios.

\section{Atividades ligadas diretamente aos usuários} Aprovada As atividades ligadas ao suporte aos usuários estão inseridas na dimensão de trabalho clínico-assistencial (BRASIL, 2014). Esta é desenvolvida a partir das demandas da população identificadas e discutidas com a eSF o que favorece o cuidado compartilhado, a clínica ampliada e a cogestão do cuidado

Segundo o monitoramento realizado pela coordenação NASF com base nos dados extraídos do e-SUS AB, em 2019 o NASF-Recife teve uma média de atendimentos individuais de 3.563 atendimentos/mês. Em 2020, nos primeiros meses, as equipes realizaram 2.499 atendimentos em Janeiro e 1.931 em Fevereiro. Com o início da pandemia e o aumento de casos, 
houve a reorganização do processo de trabalho da Atenção Primária.

Mesmo com a redução das atividades individuais devido aos protocolos de segurança, os atendimentos diretos aos usuários permaneceram em algumas situações de urgência que precisaram de suporte das equipes NASF, bem como os atendimentos aos usuários que já estavam em acompanhamento. No período de março a abril de 2020 tivemos um total de 1.262 atendimentos individuais, sendo 313 destes realizados nos domicílios e 1.355 atividades coletivas. Deste quantitativo podemos observar que o maior número de atendimentos realizados foi para os adultos do sexo feminino, conforme Gráfico 1.

Figura 02 - Número absoluto de atendimentos individuais realizados pelo NASF-Recife por faixa etária.

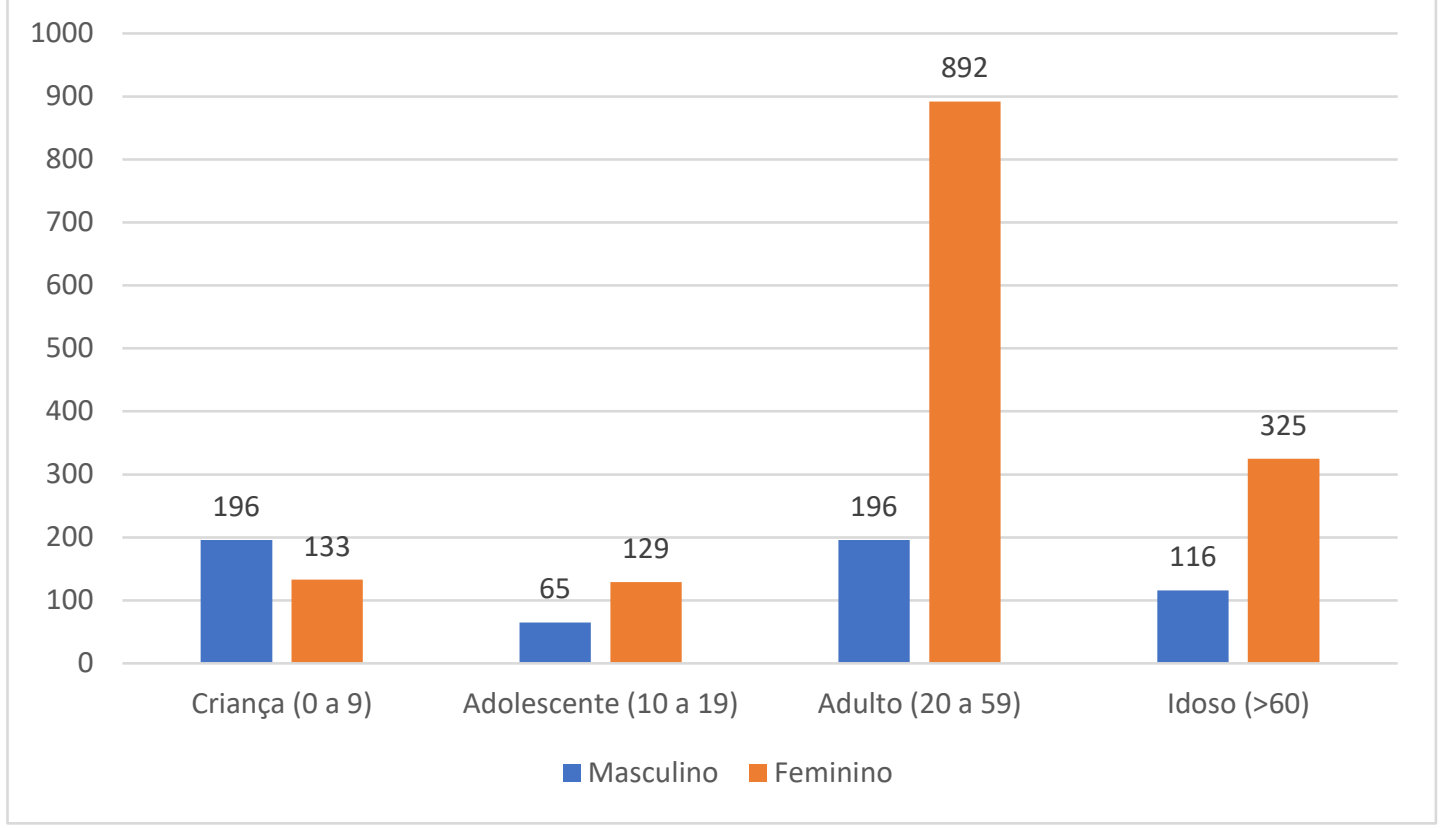

Fonte: Dados extraídos do e-SUS AB em 20 de Maio de 2020.

O processo desses atendimentos foi repensado com o cuidado em garantir a segurança necessária e, por isso, os atendimentos remotos, via telefone e pelo aplicativo WhatsApp, passaram a fazer parte do escopo de possibilidades de intervenção do profissional NASF. Algumas equipes propuseram a criação de grupos no WhatsApp em cinco linhas: a atenção ao ciclo gravídico-puerperal, atenção à saúde mental, atenção à saúde da criança, atenção à saúde do idoso e atenção em reabilitação. Através da entrada nos grupos e da necessidade de cada usuário serão realizadas estratégias como o monitoramento telefônico e/ou presencial e o repasse de materiais educativos elaborados pela equipe para cada grupo.
Com relação as demandas dos atendimentos remotos, tiveram como destaque: a) as questões de saúde mental, como ansiedade e transtornos do pânico, atrelados ao momento vivido de pandemia pela COVID-19 e suporte ao manejo do luto por perda de familiares e pessoas próximas; b) questões da assistência social ligadas aos benefícios como auxílio emergencial do governo federal, bolsa família, Benefício de Prestação Continuada e a facilitação na aquisição de documentos e condução de casos que necessite de intervenção para direitos violados de usuários do território; c) questões específicas e urgentes no cuidado com a reabilitação. 
Outra estratégia de aproximação com os usuários tem sido as lives em redes sociais realizadas pelos profissionais, em especial os Psicólogos. Durante uma pandemia é esperado que estejamos frequentemente em estado de alerta, preocupados, confusos, estressados e com sensação de falta de controle frente às incertezas do momento, por isso as ações ligadas a Saúde Mental foram temas de várias dessas iniciativas.

Além disso, uma série de materiais educativos foram elaborados nesse período seja pelas equipes com orientações gerais sobre a Pandemia e cuidados preventivos necessários, seja pelo profissional com informações direcionadas de sua categoria de formação. As temáticas foram em torno de orientações de cuidados em saúde mental, alimentação saudável e imunidade, organização da rotina, manejo do sono, sugestões de atividades e brincadeiras para crianças, informações para gestantes e puérperas, orientações para cuidadores de acamados e pessoas com deficiência.

\section{Suporte às equipes de Saúde da Família}

No que se refere ao suporte às eSF, cada eNASF focou nas demandas específicas do processo de trabalho de cada equipe apoiada através da representação do técnico de referência em cada Unidade de Saúde da Família (USF). O que, para além de manter a cobertura, facilitar a comunicação e o planejamento, contribuiu para a não aglomeração, minimizando o risco de exposição e uso racional dos EPIs.

Em 23 de Março houve o início antecipado da campanha Nacional de Vacinação contra a influenza a qual foi dividida em momentos com o escalonamento das categorias alvo (idosos, crianças, gestantes e profissionais de saúde). Esta ação demandou forte esquema logístico para assegurar a vacinação da população e ao mesmo tempo evitar aglomerações. Assim, em Recife foi montado postos volantes e polos Drive

1 Oferta orientações virtuais e permite uma classificação de risco do paciente e, se necessário,
Thru, além das salas de vacina das unidades de saúde.

O NASF participou no apoio durante toda a campanha de vacinação da gripe, seja nas USF, nos domicilio durante visita a usuários acamados e também nos postos volantes, como creches e escolas, no intuito de auxiliar na organização das filas, nas orientações aos usuários acerca da doença e dos meios de prevenção, como a correta lavagem de mãos, a etiqueta respiratória, uso e manuseio da máscara e a importância do distanciamento social.

Posteriormente, com o aumento de casos de COVID-19 nos territórios, também houve inserção dos profissionais NASF: a) no processo de acolhimento e/ou triagem dos usuários nas unidades de saúde; b) organização do fluxo interno da unidade; c) participação na equipe de telefonia, para monitoramento dos casos sintomáticos; entre outros.

Sobre a inserção dos profissionais no monitoramento, com a abertura das unidades de referência aos casos suspeitos de COVID-19 e a implantação do aplicativo web "Atende em Casa"1, as eNASF passaram a atuar de forma periódica no telemonitoramento aos usuários sintomáticos da COVID-19 via contato telefônico, atentando para as situações de complicação, agravamento do caso e que necessite de encaminhamento para outros níveis do sistema de saúde.

Outras ações do NASF foram as atividades de educação em saúde nas USF. Realizamos matriciamentos com as temáticas de "Medicamentos utilizados no combate à covid19, novas normatizações e os riscos da automedicação"; "Benefício Emergencial em período de pandemia"; cuidado com a saúde e medidas preventivas de "etiqueta respiratória"; e confecção e divulgação de material informativo sobre atendimentos online.

uma vídeochamada (teleorientação) com enfermeiros ou médicos, sobre a Covid-19. 
Nas salas de espera as temáticas abordadas foram relacionadas à: "como manter a saúde mental em tempos de distanciamento social"; "Orientações sobre condutas de prevenção à COVID-19: uso e manuseio adequados de máscara e higienização das mãos"; "distanciamento social no dia a dia"; "Concessão do benefício emergencial", e outros temas relevantes ao objetivo da quebra da cadeia de transmissão no município, entendendo que grande parte da população pode ser assintomática.

Nesse sentido, pontuamos a necessidade de reinventar com segurança e responsabilidade sanitária os momentos e espaços para estas atividades otimizando em sala de espera, triagem, sala de vacina, filas aos redores das unidades de saúde.

Vale destacar que além das atividades nas eSF, a inquestionável mudança na rotina dos profissionais de saúde devido ao enfrentamento ao novo coronavírus, que por vezes precisaram se afastar de seus familiares, gerou uma necessidade de olhar cuidadoso e ações destinadas a esses profissionais. Assim, a eNASF não hesitou em ofertar uma escuta qualificada e acolhedora, suporte emocional, terapias convencionais e práticas integrativas visando amenizar os sintomas de dor, ansiedade, medo e formas de enfrentamento ao isolamento social pela COVID-19.

Essas ações de suporte foram realizadas através de encontros grupais (preconizando as medidas de segurança) nos quais os profissionais compartilham suas expectativas e sentimentos diante do processo da pandemia; suporte individual psíquico emocional por meio telefônico; e, confecção de materiais como o "Jornal das Flores" com receitas, dicas de atividades físicas, sugestão de filmes e informações sobre os profissionais das equipes de Saúde da Família. O Jornal ainda conta com a sessão "O que vi de bom" traz tudo de positivo que aconteceu na semana e homenagens aos profissionais que se recuperaram da COVID-19 e retornaram as atividades.

\section{As ações nos territórios}

As ações nos territórios são importantes estratégias de cuidado e vínculo com os usuários e nesse período teve como objetivo difundir informações confiáveis adaptadas ao contexto das comunidades e contribuir para prevenir o contágio entre esses grupos sociais.

As ações educativas foram direcionadas a prevenção e enfrentamento a COVID-19 nas ruas dos bairros, próximo a comércios e feiras livres com o objetivo de informar sobre a importância do distanciamento social, etiqueta respiratória, o que é a quarentena, os cuidados com a saúde e demais medidas preventivas. Essas atividades foram desenvolvidas de diferentes formas, seja caminhando pela comunidade ou dentro do carro expondo música temática e avisos via microfone.

Algumas unidades realizaram o formato de cinema na comunidade, onde foi projetado imagens de orientação e esclarecimento, de como prevenir a COVID-19, e o papel importante da atuação dos profissionais das USF. Outras USF, junto com as eNASF, confeccionaram e distribuíram máscaras de tecidos para comunidade, disponibilizando também orientações sobre uso e higienização.

Sob a perspectiva do território vivo (2002) as ações de saúde de promoção e/ou prevenção em âmbito territorial estão sendo pensadas a partir de uma compreensão de sua dinâmica interna e da análise da situação epidemiológica específica em que vivemos.

\section{CONSIDERAÇÕES FINAIS}

O enfrentamento à pandemia do novo coronavírus tem sido, sem dúvida, um enorme desafio para a atuação da saúde pública do Brasil e no mundo. Com isso, vemos a importância do fortalecimento do SUS em todos os seus componentes: vigilância, cuidado em todos os níveis de assistência (primário, secundário e terciário), pesquisa e extensão. 
Nesse sentido, a Atenção Primária à Saúde brasileira com as equipes de saúde da família, que possuem o papel de ordenadora do cuidado e atuam com o enfoque territorial, que vem mudando a realidade sanitária do país nas últimas décadas. Hoje exerce um papel fundamental para o impacto positivo na saúde da população contribuindo para a abordagem comunitária essencial no enfrentamento da pandemia. Enquanto equipe de apoio a esse cuidado no território, o NASF se apresenta mais uma vez como uma estratégia potente de cuidado, uma vez que está próximo à população e suas vulnerabilidades.

Assim, em quase três meses de enfrentamento ao coronavírus percebemos o quanto o alinhamento e trocas entre gestão e trabalhadores se faz necessário. Neste movimento de avaliação e monitoramento, podemos observar que o NASF Recife continua o seu fazer de forma responsável e colaborativa.

Com o início da Pandemia percebeu-se uma dificuldade de compreensão da própria eSF sobre a atuação da eNASF nesse momento, pois os profissionais eram inicialmente questionados sobre sua permanência/atuação nas USF, uma vez que, era necessário garantir a ocupação de poucos profissionais e usuários, evitando assim, a aglomeração desnecessária. Essa dificuldade foi minimizada, com a separação das US entre referência de COVID-19 e de atendimento de rotina, fincando o NASF de forma, mais recorrente, nas unidades de rotina, dando continuidade ao seu processo de trabalho para além das ações de enfrentamento à COVID-19 (RECIFE, 2020d).

O medo de contaminação é algo que perpassa todo trabalhador da linha de frente e isso não foi diferente com os profissionais NASF. Por isso, consideramos os turnos de atividades remotas $\mathrm{e}$ para os trabalhadores do grupo de risco o processo de trabalho foi organizado para que a circulação fosse menor entre as unidades.

Outros desafios como o esvaziamento dos usuários das unidades de saúde, o afastamento dos profissionais das equipes apoiadas seja por estar no grupo de risco ou por adoecimento, fizeram com que os profissionais NASF precisassem reajustar, constantemente, seu próprio processo de trabalho. Diante disso, as NT traziam mais clareza nas modalidades e possibilidades de atuações para esse momento, porém deixando em aberto a oferta de práticas diferentes das apontadas, respeitando-se a singularidade de cada território.

Destacamos que o vínculo já existente entre profissionais das eSF e eNASF facilitou essa adaptação, bem como a atitude proativa e disponibilizada das eNASF, por entender, o impacto do seu trabalho frente a pandemia e a necessidade da continuidade do atendimento aos pacientes crônicos acompanhados anteriormente. Diante disso, ações foram pensadas e executadas, visando a manutenção do vínculo entre as equipes, o cuidado a esses trabalhadores e a comunidade.

Como resultado dessas inúmeras atividades lançamos, em maio, um Boletim informativo do NASF Recife como um instrumento de divulgação das ações realizadas no início da pandemia até os dias atuais. Assim também, reconhecemos a contribuição dada por cada profissional NASF que, diariamente, pode, por meio do seu trabalho, fortalecer o combate ao coronavírus e o trabalho da APS.

No momento atual não temos dados para mensurar a efetividades das ações do NASF em relação as equipe e usuários que não recebem apoio. Entretanto, podemos afirmar a importância do trabalho focado no território e a proximidade com as realidades das equipes de saúde da família, pois no dia a dia do processo de trabalho percebemos que o NASF tem sido cada vez mais demandado pelas equipes, o que pode indicar, um reconhecimento da potência do trabalho no território, bem como a necessidade de expansão das eNASF potencializando todas as eSF do município.

Ainda não temos como avaliar a extensão temporal na qual se dará a pandemia no Brasil, 
nem os próximos enfrentamentos que serão necessários. Entretanto, atuar na APS do Recife no combate à COVID-19, torna-se uma oportunidade de aprendizagem em variados aspectos. A possibilidade de atuação em diversas atividades presenciais ou virtuais, gera tanto nos profissionais quanto nos gestores um crescimento profissional e pessoal, ampliando a capacidade de visão da comunidade e o desenvolvimento de diferentes habilidades para o enfrentamento deste desafio e outros tantos que virão no âmbito da promoção, prevenção e intervenção à saúde. Além de reafirmar a força de uma APS forte e resolutiva com o foco das suas equipes na atuação em diálogo com os territórios. 


\section{NOTAS E REFERÊNCIAS}

BRASIL. Ministério da Saúde. Secretaria de Atenção à Saúde. Departamento de Atenção Básica. Núcleo de Apoio à Saúde da Família/Ministério da Saúde, Secretaria de Atenção à Saúde. Departamento de Atenção Básica. Brasília: Ministério da Saúde, 2014. 112p. (Cadernos de Atenção Básica, no. 39).

BRASIL.Ministério da Saúde. Portaria no 2.436, de 21 de Setembro de 2017. Aprova a Política Nacional de Atenção Básica, estabelecendo a revisão de diretrizes para a organização da Atenção Básica, no âmbito do Sistema Único de Saúde (SUS). Brasília: Ministério da Saúde; 2017.

BRASIL. Ministério da Saúde. Secretaria de Vigilância em Saúde. Painel de casos de doença pelo coronavírus 2019 (COVID-19) no Brasil pelo Ministério da Saúde. Brasília, 2020. Disponível em:< https://covid.saude.gov.br >Acesso em: 28 mai. 2020.

Campos GWS, Domitti AC. Apoio matricial e equipe de referência: uma metodologia para gestão do trabalho interdisciplinar em saúde. Cad Saúde Pública. 2007;23(2):399-407.

Merhy, E. E. Saúde: cartografia do trabalho vivo. São Paulo: Hucitec, 2002.

Secretaria de Saúde. Diretoria Executiva de vigilância à Saúde. Boletim Epidemiológico. N. 72, Ano 2020, Recife. Disponível em:< https://cievsrecife.files.wordpress.com> Acesso em: 25 mai. 2020a.

Secretaria de Saúde. Diretora Executiva de Atenção Básica e Gestão Distrital. Coordenação Núcleo de Apoio à Saúde da Família. Nota Técnica 01. Processo de trabalho do NASF no contexto de enfrentamento da emergência em saúde pública decorrente do novo coronavírus (COVID-19). N. 01, Recife. 2020 b.

Secretaria de Saúde. Diretora Executiva de Atenção Básica e Gestão Distrital. Protocolo de Assistência e Manejo Clínico do novo Coronavírus (COVID-19) na APS do município de Recife. N. 01, Ano 2020. Recife, 2020c.

Secretaria de Saúde. Diretora Executiva de Atenção Básica e Gestão Distrital. Protocolo de Assistência e Manejo Clínico do novo Coronavírus (COVID-19) na APS do município de Recife. N. 02, Ano 2020. Recife, 2020d.

Secretaria de Saúde. Diretora Executiva de Atenção Básica e Gestão Distrital. Coordenação Núcleo de Apoio à Saúde da Família. Nota Técnica 02. Processo de trabalho do NASF no contexto de enfrentamento da emergência em saúde pública decorrente do novo coronavírus (COVID-19). N. 01, Recife. 2020e.

Secretaria de Saúde. Diretora Executiva de Atenção Básica e Gestão Distrital. Protocolo de Assistência e Manejo Clínico do novo Coronavírus (COVID-19) na APS do município de Recife. N. 03, Ano 2020. Recife, $2020 f$. 